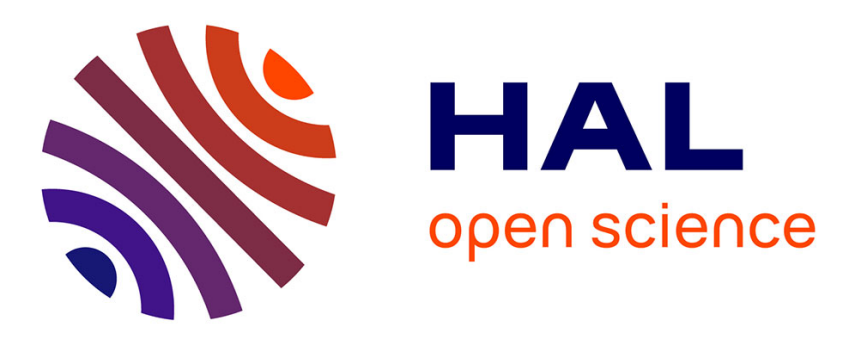

\title{
La perception du migrant et de l'intégration dans l'opinion française: confusions et stéréotypes
}

Sylvain Brouard, Vincent Tiberj

\section{To cite this version:}

Sylvain Brouard, Vincent Tiberj. La perception du migrant et de l'intégration dans l'opinion française : confusions et stéréotypes. Hommes \& migrations, 2006, Accueillir autrement, 1261, pp.120-130. 10.3406/homig.2006.4471 . halshs-00572411

\section{HAL Id: halshs-00572411 https://shs.hal.science/halshs-00572411}

Submitted on 25 Mar 2019

HAL is a multi-disciplinary open access archive for the deposit and dissemination of scientific research documents, whether they are published or not. The documents may come from teaching and research institutions in France or abroad, or from public or private research centers.
L'archive ouverte pluridisciplinaire HAL, est destinée au dépôt et à la diffusion de documents scientifiques de niveau recherche, publiés ou non, émanant des établissements d'enseignement et de recherche français ou étrangers, des laboratoires publics ou privés. 


\section{La perception du migrant et de l'intégration dans l'opinion française : confusions et stéréotypes \\ Sylvain Brouard, Vincent Tiberj}

\section{Résumé}

Que ce soit autour de l'islamophobie et de son éventuelle montée dans l'opinion, de la nature même de la République française et de son projet, ou de l'association entre immigration et délinquance, notre modèle d'intégration pose question. Quelle est la position des Français dans ces débats, quelles logiques suivent-ils ? Analyse à partir de deux enquêtes simultanées, réalisées en avril 2005, auprès d'un échantillon représentatif des "nouveaux Français" et de la population française de 18 ans et plus.

\section{Citer ce document / Cite this document :}

Brouard Sylvain, Tiberj Vincent. La perception du migrant et de l'intégration dans l'opinion française : confusions et stéréotypes. In: Hommes et Migrations, n¹261, Mai-juin 2006. Accueillir autrement. pp. 120-130;

doi : https://doi.org/10.3406/homig.2006.4471

https://www.persee.fr/doc/homig_1142-852x_2006_num_1261_1_4471

Fichier pdf généré le 27/02/2019 


\section{La perception du migrant et de l'intégration dans l'opinion française : confusions et stéréotypes}

Que ce soit autour de l'islamophobie et de son éventuelle montée dans l'opinion. de la nature même de la République française et de son projet, ou de l'association entre immigration et délinquance, notre modèle d'intégration pose question. Quelle est la position des Français dans ces débats. quelles logiques suivent-ils? Analyse à partir de deux enquêtes simultanées, réalisées en avril 2005, auprès d'un échantillon représentatif des "nouveaux Français" et de la population française de 18 ans et plus.

Par Sylvain Brouard et Vincent Tiberj, Cevipof / Sciences Po. Auteurs de Français comme les autres?
1)- Voir notamment, Vincent Geisser, La nouvelle islamophobie, La Découverte, Paris, 2003, ou Thomas Deltombe, LIslam imaginaire : la construction médiatique de l'islamophobie en France, 1975-2005, La Découverte, Paris, 2005.

2)- Pascal Blanchard, Nicolas Bancel et Sandrine Lemaire (dir.), La Fracture coloniale, La Découverte, Paris, 2005.
"Les Français s'habituent aux idées du Front national". C'est ainsi que, le 15 décembre 2005, Le Monde titrait en une à propos des résultats d'une enquête TNS-Sofrès. De fait, le contexte autour de l'immigration, particulièrement quand celle-ci s'apparente aux mouvements de population venant du Maghreb, semble bien s'être tendu dans l'Hexagone. Il paraît loin le temps où la France se glorifiait d'être plurielle au lendemain de la victoire de la Coupe du monde, en 1998. Aujourd'hui, c'est la capacité de la France à se vivre comme diverse qui est en question etsa capacité à intégrer de nouvelles populations, nouvelles en tout cas par le ressenti qu'elles suscitent alors que les petits-enfants de l'immigration maghrébine commencent à atteindre leur majorité.

La mise en cause du modèle d'intégration français emprunte à des registres différents. Pour certains, cette difficulté à intégrer viendrait de valeurs et d'attitudes trop éloignées du modèle "républicain", valeurs associées souvent à une religion, l'islam. Cette religion est d'ailleurs bien ressentie comme l'une des clefs médiatiques et politiques de lecture des difficultés d'intégration des nouveaux arrivants en France : que l'on se remémore les interrogations autour du 11Septembre et sur notre capacité à créer un islam de France, les tensions entre voile et laïcité, et plus récemment les débats autour des caricatures de Mahomet. Les questions autour de l'islamophobie et de son éventuelle montée dans l'opinion doivent donc être posées ${ }^{(1)}$.

Lintégration à la française est également remise en question par une sorte de retour du refoulé historique, véhiculée par des associations aux projets et positions antagonistes autour de la question des mémoires de l'histoire coloniale, de la guerre d'Algérie ou de l'escla$\operatorname{vage}^{(2)}$. Par son histoire, c'est la nature même de la République fran- 
çaise et de son projet qui est en débat, sans même évoquer les discussions et les débats autour de la loi du 23 février 2005.

Lintégration est redevenue l'une des problématiques de la question de l'immigration liee aux relations entre immigration et chômage, longtemps mise en avant par la droite de la droite. Lidée de l'immigration comme cause du chômage progresse dans de nouveaux segments de la population et concerne de nouveaux flux : en premier lieu, l'immigration attendue d'Europe de l'Est ${ }^{(3)}$. Lors de la campagne référendaire de 2005, les menaces pour la pérennité des emplois causées par l'élargissement de l'Union européenne expliquent le succès de la thématique du plombier polonais, à propos de laquelle il est difficile de négliger la part de rejet du migrant. L'association entre immigration et délinquance ${ }^{(4)}$, que cette association soit fantasmée ou réelle, est une autre dimension de l'appréhension de la question de l'intégration. A cet égard, entre la dénonciation de la polygamie et la proposition d'expulser les étrangers émeutiers, les discours sur les événements de novembre 2005 en banlieue sont particulièrement éclairants.
Abdellah Ed D., Maroc, vit en Italie depuis 20 ans et est depuis septembre 2005 en France. 
5)- Ronald Inglehart, Culture shift in advanced industrial society, Princeton, Princeton university Press, 1990.

6)- Voir notamment, Nonna Mayer, Ces Français qui votent Le Pen, Paris, Flammarion, 2002 ou Etienne Scwesiguth, "La montée des valeurs individualistes", Futuribles, (200), juillet-aout 1995 : p. 131-160.

7)- 0. Duhamel, "Révolte des banlieues et image du Front national", in 0. Duhamel \& B. Teinturier, TNS SOFRES L'état de l'opinion 2006, Paris, 2006.

8)- Gerard Noiriel, Le creuset français : histoire de l'immigration, $19^{2}$-20 siècles, Seuil, Paris, 1988.

9)- Pour un descriptif de la méthode, ce type d'enquête auprès des populations d'origine immigrée ayant souvent été remis en question, voir Sylvain Brouard \& Vincent Tiberj, Français comme les autres? Enquête sur les citoyens d'origine maghrébine, africaine et turque, Presses de Sciences Po, Paris, 2005.
Le modèle d'intégration est incontestablement en question. Comment les Français se positionnent-ils dans ces débats et selon quelles logiques?

La France est-elle dans une phase de raidissement à l'égard des immigrés ou bien suit-elle le mouvement vers plus de tolérance, conformément au modèle du postmatérialisme de Ronald Inglehart ${ }^{(5)}$ ? Après tout, les chercheurs spécialistes des opinions ethnocentristes remarquent depuis plusieurs décennies que les nouvelles générations sont systématiquement plus ouvertes que celles qui les ont immédiatement précédées $^{(6)}$. Si c'est le raidissement qui prime, est-ce une forme de banalisation des préjugés anti-immigrés ou une lepenisation (voir Olivier

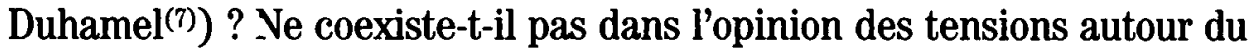
modèle d'intégration oscillant entre le développement d'une société multiculturelle ou d'un modèle assimilationniste ? Qu'attend l'opinion des immigrés et de leurs enfants ? Qu'ils se fondent dans la masse d'un "mainstream" à la française dont il est somme toute difficile de déterminer les contours ? Ou bien qu'ils viennent enrichir de leurs particularités "un creuset" à la française selon l'expression de Gérard Noiriel ${ }^{(8)}$ ?

\section{L'intégration : un état des lieux}

Pour répondre à ces questions nous nous fonderons sur deux enquêtes simultanées, réalisées en avril 2005, l'une menée auprès d'un échantillon représentatif des "nouveaux Français" (immigrés naturalisés, première et deuxième génération nées en France) d'origine maghrébine, africaine et turque, l'autre représentative de la population française de 18 ans et plus ${ }^{(9)}$. Lintérêt de cette comparaison réside dans les différences de jugement entre les deux échantillons et les logiques qui peuvent les produire,

Tableau $n^{\circ} 1$ : Les jugements sur la situation de l'intégration

\begin{tabular}{l|c|c} 
& $\begin{array}{c}\text { Enquête Rapfi } \\
\text { nouveaux } \\
\text { Français }\end{array}$ & $\begin{array}{c}\text { Enquête "miroir" } \\
\text { ensemble } \\
\text { de l'électorat }\end{array}$ \\
\hline $\begin{array}{l}\text { Dans les prochaines années, la situation des immigrés } \\
\text { va se détériorer (\%) }\end{array}$ & $27 \%$ \\
$\begin{array}{l}\text { Il y a trop d'immigrés en France (\% d'accord) } \\
\begin{array}{l}\text { En France, tout le monde peut réussir quelle que soit } \\
\text { la couleur de sa peau (\% d'accord) }\end{array}\end{array}$ & $37 \%$ \\
$\begin{array}{l}\text { Les immigrés peuvent s'intégrer facilement (\%) } \\
\begin{array}{l}\text { A propos de l'intégration, ce sont avant tout les personnes } \\
\text { d'origine étrangère qui ne se donnent pas les moyens } \\
\text { de s'intégrer (\%) }\end{array}\end{array}$ & $49 \%$ \\
$\begin{array}{l}\text { À propos de l'intégration, c'est avant tout la société } \\
\text { française qui ne donne pas les moyens aux personnes d'origine } \\
\text { étrangère de s'intégrer (\%) }\end{array}$ & $41 \%$ & $33 \%$ \\
\hline
\end{tabular}


entre le jugement porté par les individus qui participent ou sont perçus comme concernés par l'intégration et le reste de la population.

On constate des variations entre les deux populations étudiées, mais d'intensité inégale. Il en va ainsi de l'avenir de la situation des immigrés en France où des proportions similaires de personnes interrogées s'avèrent pessimistes ou optimistes, mais est-ce pour les mêmes raisons ? D'une manière générale, les personnes issues de l'immigration maghrébine, africaine ou turque sont plus confiantes sur les capacités d'intégration des migrants ( $41 \%$ considèrent qu'ils peuvent s'intégrer très ou assez facilement contre $33 \%$ dans l'ensemble de la population), ils sont également $49 \%$ à considérer la société française comme "aveugle

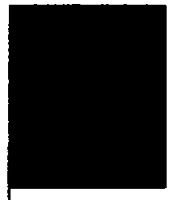
L'intégration prend aujourd'hui dans l'électorat une dimension religieuse, faisant ainsi écho aux débats autour de l'islam, qui ont marqué la période récente. aux couleurs", soit 6 points de plus que pour les Français en général ; en effet, parmi les nouveaux Français, le niveau de chômage et de précarité est près du double de celui constaté dans l'enquête miroir ${ }^{(10)}$. Enfin, les nouveaux Français s'avèrent moins sujets au préjugé anti-immigré que l'électorat en général (37\% contre $47 \%$ ), même si cette attitude est due plutôt à leur appartenance plus fréquente aux jeunes générations en France et à un niveau de diplôme plus élevé qu'à leur origine familiale. Dans l'échantillon représentatif de l'ensemble des Français, une logique particulière se fait jour autour du facteur religieux. Considérer ou non qu'il y a trop d'immigrés ne fait pas varier l'appréciation de la capacité d'intégration des immigrés (entre $30 \%$ et $35 \%$ considèrent qu'ils peuvent s'intégrer facilement). En revanche, l'attitude à l'égard de l'islam entraîne de fortes variations : quand l'islam a une image très positive, $48 \%$ des personnes interrogées considèrent que l'intégration est facile, quand elle est très négative, la proportion n'est plus que de $28 \%$. L'intégration prend donc aujourd'hui dans l'électorat une dimension religieuse, faisant ainsi écho aux débats autour de l'islam, qui ont marqué la période récente.

\section{La responsabilité des difficultés d'intégration}

Les différences entre les deux échantillons sont maximales quant à l'explication des difficultés d'intégration, laissant apparaître une réelle tension sur ce sujet. Les causes des difficultés de l'intégration sont imputées de manière inverse. La responsabilité incombe avant tout à la société (59\%) pour les nouveaux Français contre $26 \%$ aux individus, alors que dans la société française dans son ensemble, le diagnostic le plus courant est d'incriminer les individus (48\%) plutôt que la société.

Cette différence persiste quand on compare les réponses à cette question par groupes sociopolitiques. Ainsi, parmi les nouveaux Français, la primauté de la responsabilité de la société se fait jour indépendamment

10)- Nos résultats sont d'ailleurs conformes à ceux obtenus par Jean-Luc Richard, voir : "Le rapport entre les niveaux de formation et la fréquence du chômage des fils et filles d'immigré (s) et les origines nationales de leurs parents", Migrations et société, à paraître, mai 2006. 
Tableau $n^{\circ} 2$ : Répondants (\%) attribuant la responsabilité des difficultés d'intégration aux individus, selon les échantillons et leur attitude vis-à-vis des immigrés et du racisme dans la société française.

\begin{tabular}{l|c|c|c|c|} 
& \multicolumn{2}{|c|}{$\begin{array}{c}\text { Enquête Rapfi } \\
\text { nouveaux Français }\end{array}$} & \multicolumn{2}{c}{$\begin{array}{c}\text { Enquête "miroir" } \\
\text { ensemble de l'électorat }\end{array}$} \\
\hline & $\begin{array}{c}\text { En France tout } \\
\text { le monde peut } \\
\text { réussir quelle que } \\
\text { soit la couleur } \\
\text { de sa peau }\end{array}$ & $\begin{array}{c}\text { Il y a trop } \\
\text { d'immigrés } \\
\text { en France }\end{array}$ & $\begin{array}{c}\text { En France tout } \\
\text { le monde peut } \\
\text { réussir quelle que } \\
\text { soit la couleur } \\
\text { de sa peau }\end{array}$ & $\begin{array}{c}\text { Il y a trop } \\
\text { d'immigrés } \\
\text { en France }\end{array}$ \\
\hline Tout à fait d'accord & $36 \%$ & $43 \%$ & $64 \%$ & $72 \%$ \\
Plutôt d'accord & $31 \%$ & $38 \%$ & $59 \%$ & $67 \%$ \\
Plutôt pas d'accord & $22 \%$ & $20 \%$ & $45 \%$ & $38 \%$ \\
Pas du tout d'accord & $17 \%$ & $18 \%$ & $29 \%$ & $21 \%$
\end{tabular}

du diplôme des personnes interrogées, de leur profession, de leur âge ou de leur relation à l'immigration (immigrés naturalisés, première et deuxième génération née en France), voire de leur alignement politique. En revanche, l'électorat dans son ensemble s'avère politiquement et socialement divisé. Les plus jeunes (30 ans et moins) mettent en avant la responsabilité de la société alors que les plus de 42 ans sont majoritairement sur la position inverse. Les titulaires d'un diplôme du supérieur mettent majoritairement l'accent sur la responsabilité de la société alors que les sans-diplômes, les titulaires d'un diplôme du primaire ou du secondaire sont sur la position inverse. Enfin, la droite invoque majoritaircment la responsabilité individuelle quand la gauche est sur la position inverse.

De surcroît, des logiques différentes sont sous-jacentes à l'imputation de la responsabilité des problèmes d'intégration. Parmi les nouveaux Français, dans tous les cas, la société est tenue plus souvent responsable des difficultés de l'intégration que les immigrés. Ainsi, même parmi les nouveaux Français qui s'avèrent les plus anti-immigrés, ils ne sont que $43 \%$ à mettre la responsabilité des problèmes d'intégration sur les personnes contre $48 \%$ qui préfèrent mettre en avant la société d'accueil et $8 \%$ à partager les responsabilités.

Dans la population française en général, les choses diffèrent. Si la société française est considérée comme aveugle aux couleurs, c'est la responsabilité des individus qui est majoritairement mise en avant. Lorsque l'effet du racisme dans la société française est reconnu, la responsabilité de la société est prépondérante. Il en va de même pour l'opinion à l'égard des immigrés et de leur nombre dans l'Hexagone, qui scinde les opinions à l'égard des responsabilités dans les problèmes d'intégration. Enfin on retrouve encore la logique religieuse qui vient s'ajouter à la logique anti-immigré : quand l'islam est vu positivement c'est la 
société qui est pointée du doigt, quand il est vu négativement, ce sont les immigrés. Lorsque les Français répondent à des questions sur l'intégration, ils répondent aussi à des questions sur l'islam et les musulmans, montrant combien l'amalgame est désormais fort, dans l'opinion publique. Lintégration n'est donc plus seulement une question économique ou politique, elle devient aussi religieuse, comme s'il fallait désormais d'abord statuer sur le statut assimilable de cette religion avant de s'intéresser aux migrants qui pourtant sont loin de tous s'en réclamer ${ }^{(11)}$.
11)- $59 \%$ des nouveaux Français se déclarent musulmans, alors que $65 \%$ des Français en général se disent catholiques.

\section{Les conceptions de l'intégration}

$\Lambda$ près avoir analysé les difficultés de l'intégration et leurs responsables pour en comprendre les déterminants, il convient d'éclairer les conceptions implicites de l'intégration qui fondent les jugements de l'opinion.

Au-delà de la question de l'intégration, se pose donc la question du modèle qui la fonde. Qu'attendent les Français des immigrés à cet égard ? Quel modèle de société souhaitent-t-ils ? Penchent-ils plutôt vers l'assimilation, entendue comme l'obligation pour les nouveaux arrivants de se conformer à un "mainstream" français, ou vers une société multiculturelle?

D’après vous, le plus important aujourd'hui est :

\begin{tabular}{|c|c|c|c|c|}
\hline & $\begin{array}{l}\text { de chercher à } \\
\text { s'intégrer en évitant } \\
\text { tout conflit avec } \\
\text { le reste de la société }\end{array}$ & $\begin{array}{l}\text { de revendiquer ses } \\
\text { droits, même si cela } \\
\text { crée des tensions }\end{array}$ & Sans réponse & Total \\
\hline Enquête Rapfi & 54 & 43 & 3 & 100 \\
\hline \multirow[t]{2}{*}{ Enquête miroir } & 65 & 32 & 3 & 100 \\
\hline & $\begin{array}{c}\text { de traiter tous } \\
\text { les Français } \\
\text { de la même manière }\end{array}$ & $\begin{array}{c}\text { de corriger } \\
\text { les effets } \\
\text { des discriminations }\end{array}$ & Sans réponse & Total \\
\hline Enquête Rapfi & 41 & 59 & 0 & 100 \\
\hline \multirow[t]{2}{*}{ Enquête miroir } & 58 & 41 & 1 & 100 \\
\hline & $\begin{array}{l}\text { de mettre en valeur } \\
\text { les différences } \\
\text { culturelles entre } \\
\text { les Français }\end{array}$ & $\begin{array}{c}\text { d'insister sur ce que } \\
\text { les Français } \\
\text { ont en commun }\end{array}$ & Sans réponse & Total \\
\hline Enquête Rapfi & 43 & 55 & 2 & 100 \\
\hline Enquête miroir & 31 & 67 & 2 & 100 \\
\hline
\end{tabular}


Les différences entre les nouveaux Français et le reste de la société sont de l'ordre des nuances plutôt que des contrastes, à une exception près. Le seul point sur lequel les deux échantillons diffèrent en effet est celui de la lutte contre les discriminations. Celle-ci est mise en avant par rapport à l'égalité de traitement que privilégie une majorité de l'échantillon miroir. On pourrait y voir la revendication ou au minimum le désir d'un traitement différencié, voire de reconnaissance de la part de la société française d'une société multicommunautaire, mais ce n'est pas la tendance générale qui se dégage parmi les nouveaux Français. Majoritairement, ils penchent du côté de l'intégration à la société française et insistent sur la communauté de destin avec le reste de la société. Néanmoins, au-delà de cette première approche grâce à une analyse de classification hiérarchique, on peut identifier plusieurs conceptions de l'intégration qui traversent les deux populations étudiées.

En partant des trois questions sur le modèle d'intégration et sur la responsabilité des difficultés d'intégration - en utilisant la méthode de la classification ascendante hiérarchique, dont l'objet est de repérer et d'identifier des profils de réponses similaires dans un échantillon - on a pu distinguer, au sein de l'électorat français, quatre groupes : les assimilationnistes ( $46 \%$ de l'échantillon), les protestataires-anxieux (8\%), les républicains ( $36 \%$ ) et les multiculturels ( $10 \%)$.

\section{Les assimilationnistes et les républicains...}

Les assimilationnistes se caractérisent par le consensus qui les rassemble autour de la question des responsabilités : c'est aux migrants de s'intégrer (100\% de leurs réponses) et ils doivent le faire sans revendication de droits spécifiques ( $78 \%$ ), dans le cadre d'une égalité de traitement $(70 \%)$ et sans prise en compte des différences culturelles (28\%). C'est également parmi eux que l'on retrouve le plus d'anti-immigres ( $67 \%$, où le niveau d'autoritarisme est le plus fort (66 \% prônent la discipline à l'école et 41 \% le rétablissement de la peine de mort), où l'attitude à l'égard de l'islam est la plus négative ( $45 \%$ de positifs contre $60 \%$ et plus dans les trois autres groupes) et l'affiliation au catholicisme la plus importante (76\%). Clairement, leur positionnement à l'égard des migrants est maximaliste et insensible à la diversité. Pour eux, il existe un modèle français et c'est aux nouveaux arrivants de s'y conformer.

Les assimilationnistes et les républicains partagent des points communs, notamment leur préférence pour le traitement égal entre les Français ( $75 \%$ ), pour l'insistance sur ce que les Français ont en commun (77\%) plutôt que le multiculturalisme et le souhait que l'intégration se fasse sans conflit ( $73 \%$ ), mais leur grande différence se situe sur les questions de responsabilité des problèmes d'intégration : ici c'est la société qui est pointée du doigt (78\% des réponses, les $22 \%$ restant répondant 
les deux). De fait, ce groupe affirme son attachement au modèle républicain d'intégration et ne rejette pas les immigrés, bien au contraire. Seuls 29 \% considèrent qu'ils sont trop nombreux en France et l'attitude négative à l'égard de l'islam est minoritaire (37\%). Ces individus sont également plutôt de gauche (48\% alors que parmi les assimilationnistes ils ne sont que $27 \%$ ), opposés à la peine de mort ( $80 \%$ ), en défaveur de la discipline à l'école (39\%). Pourtant, en filigrane, se dessine, comme pour les assimilationnistes, la volonté que les migrants s'intègrent à un modèle français et qu'ils partagent des valeurs communes qui fondent la communauté nationale hexagonale. Cependant, le modèle français n'a pas le même contenu pour ces deux groupes. Les différences en termes d'opinions sur l'autorité, la peine de mort ou sur l'islam démontrent

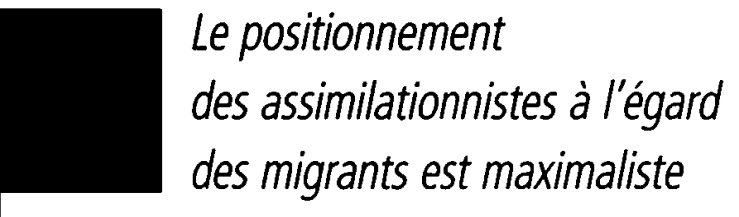
et insensible à la diversité. Pour eux, il existe un modèle français et c'est aux nouveaux arrivants de s'y conformer. bien, du côté des républicains, un idéal de société proche du postmatérialisme, mêlant ouverture et tolérance à l'égard des conduites privées mais neutralité républicaine quand il s'agit des groupes ou de la sphère publique. Au contraire, les assimilationnistes sont plus souvent du côté fermé en matière privée et proche d'un modèle de société traditionnelle, où le principe d'autorité prime. Ensuite, autant les assimilationnistes considèrent que ce sont aux migrants et aux migrants seuls de s'intégrer, autant les républicains reconnaissent les difficultés et la responsabilité de la société dans le processus d'intégration. Dès lors, les seconds sont beaucoup plus en faveur de politiques volontaristes à l'égard des immigrés que les premiers. $30 \%$ des républicains considèrent que l'état devrait aider à la construction de mosquées contre $16 \%$ des assimilationnistes, ils sont $70 \%$ à vouloir accorder plus de moyens aux écoles avec une forte proportion de familles immigrés et $51 \%$ des quotas de logement pour les familles immigrées contre $48 \%$ et $36 \%$ chez les assimilationnistes.

\section{... les multiculturels et les protestataires-anxieux}

Les multiculturels constituent un groupe à la fois spécifique et proche des républicains. Ils leur sont proches sur la question de la responsabilité des problèmes d'intégration pour lesquels la société d'accueil est mise en avant ( $86 \%$ ). Ils partagent aussi le refus du rétablissement de la peine de mort (75\%) ou de la discipline à l'école (38\%) contre l'apprentissage de l'esprit critique, une attitude positive à l'égard de l'islam (62\%) et de l'immigration (à peine $25 \%$ répondent qu'ils sont trop nombreux en France). Comme pour les républicains, la gauche est le premier groupe politique ( $53 \%$ ) et les titulaires d'un baccalauréat ou d'un diplôme supérieur sont très majoritaires $(63 \%)^{(12)}$. Mais le modèle d'in-

12)- In trait spẹcifique des multiculturels est leur âge moyen, le plus faible des quatre groupes (39 ans contre 46 pour les républicains et 50 pour les assimilationnistes). 
tégration des multiculturels diffère : ils se partagent à quasi-égalité entre la légitimité de revendications de droits spécifiques pour les immigrés et leurs enfants et la volonté d'une intégration sans conflit. Ils considèrent à $100 \%$ qu'il faut lutter contre les discriminations plutôt que de traiter tous les Français de la même manière et considèrent à 100 \% plus important de mettre en valeur les différences culturelles que ce que les Français ont en commun. Avec ce type de profil, il n'est pas surprenant de retrouver les multiculturels du côté des soutiens à l'égard de politiques volontaristes d'intégration : 81 \% sont en faveur de moyens supplémentaires pour les écoles à forte proportion de familles immigrées, $81 \%$ sont en faveur de quotas de logement pour les familles immigrées. Ils sont également $58 \%$ à être favorables à des quotas d'emploi pour les immigrés dans les entreprises (contre $44 \%$ à l'échelle de l'échantillon miroir) et $55 \%$ en faveur d'une intervention de l'État pour la construction de mosquées. Leur modèle est donc celui d'une société qui reconnaît la diversité, et dans la sphère privée et dans la sphère publique, ce qui les différencie de la neutralité publique des républicains.

Enfin, le dernier groupe, celui des protestataires-anxieux, est le plus difficile à cerner en termes de modèle d'intégration, car ils partagent des points communs avec chacun des trois groupes précédemment mentionnés. Ils sont, sur les valeurs de tolérance et de rapport à l'autorité, dans un entre-deux, partageant avec les assimilationnistes un plus fort soutien au rétablissement de la peine de mort (41\%), s'avérant plus anti-immigrés que les républicains et les multiculturels (44\% de trop d'immigrés contre $67 \%$ chez les assimilationnistes et moins de $30 \%$ dans les deux autres groupes). Ils partagent les alignements politiques des républicains et le niveau moyen de diplômes des 
assimilationnistes. Ils mettent cependant plus souvent en avant la responsabilité des immigrés dans les difficultés d'intégration, et sont unanimes sur l'accent qui doit être mis sur ce que les Français ont en commun plutôt que sur leurs différences culturelles. Cependant, ils sont tout autant pour la correction des discriminations, plutôt que pour le traitement égal, et pour la revendication des droits, plutôt que pour l'intégration sans heurt. En fait, on peut se demander si ce groupe se caractérise vraiment par une position spécifique sur l'intégration. Il semblerait au regard d'autres indicateurs dont nous disposons dans l'enquête que ce soit la culture de la protestation qui les caractérise, doublée d'un niveau de crainte économique forte.

\section{Un troisième groupe, celui des intégrationnistes}

La population française est donc traversée part des conceptions différentes, voire parfois antagonistes du modèle d'intégration, même si l'essentiel des débats se joue entre assimilationnistes et républicains, avec notamment la question des politiques publiques qui doivent être mises en place et celle de l'effort que la société française qui doit consentir ou non pour permettre une intégration réussie. Les multiculturels restent numériquement faibles. Parmi les nouveaux Français, les équilibres et les conceptions de l'intégration sont différents. Deux groupes se retrouvent parmi les Français d'origine maghrébine, africaine et turque : les assimilationnistes (13\% de l'échantillon) et les multiculturels (35\%, soit une proportion trois fois supérieure à celle constatée dans l'enquête miroir). Peu ou prou, les profils se reproduisent dans l'enquête Rapfi, que ce soit en termes de positionnement quant aux responsabilités des problèmes de l'intégration, et des conduites qu'il convient de privilégier ou des attitudes à l'égard de l'immigration ou de l'autorité.

Un troisième groupe spécifique émerge cependant, celui des intégrationnistes (44\% des personnes interrogées). Leurs traits spécifiques sont un équilibre entre la revendication de droits spécifiques et celle d'une intégration sans heurt, avec une responsabilité de la société majoritaire dans les problèmes d'intégration (77\%), obligeant à mettre en place des politiques de lutte contre les discriminations (63\%) plutôt qu'un traitement égal. Mais cette revendication d'un traitement différencié va de pair avec une unanimité en faveur de ce que les Français ont en commun, autrement dit, ce n'est pas une demande communautariste qui s'exprime dans ce groupe, mais bien la volonté de rejoindre la perception d'un mainstream hexagonal.

Le soupçon de "communautarisme" pourrait venir à l'esprit au sujet des nouveaux Français multiculturels. Cependant, cette interprétation semble difficile à soutenir(13). Ils se sentent en effet aussi proches des Français en général que les assimilationnistes de l'enquête Rapfi ( $86 \%$ contre 84 \%). Le phénomène se reproduit à l'égard
13)- Ce constat corrobore nos conclusions sur le communautarisme en France. Voir Sylvain Brouard, Vincent Tiberj, Français comme les autres?, op. cit. 
14)- Voir Sylvain Brouard, Vincent Tiberj, Français comme les autres?, op. cit. de la proximité religieuse ( $70 \%$ contre $67 \%$ ). $81 \%$ des multiculturels de l'enquête Rapfi ont une attitude positive à l'égard de la laïcité ( $83 \%$ chez les assimilationnistes). Quand il s'agit de la République, l'attitude positive rassemble $87 \%$ des multiculturels et des assimilationnistes issus de l'immigration. Enfin dans tous les groupes, quand il s'agit de la scolarisation souhaitée pour les enfants, c'est une école publique sans éducation religieuse qui recueille l'essentiel des suffrages (70\% chez les multiculturels, $72 \%$ chez les intégrationnistes, $73 \%$ chez les assimilationnistes). L'essentiel des multiculturels s'accorde donc sur les éléments constitutifs du vivre ensemble hexagonal.

$\mathrm{Au}$ final, la divergence des modèles d'intégration au sein de la société française constitue le fondement des tensions autour du modèle français. Ces différents "modèles de France" - une France melting-pot, une France républicaine et une France qui pourrait être qualifiée "d'éternelle" par certains de ceux qui la défendent - accordent une place variable à la question de la religion. Pour les tenants de ce dernier modèle, la religion devient sensible à tel point qu'ils en font un des éléments centraux d'explication de ce qu'ils perçoivent comme une panne de la machine à intégrer. L'exposition médiatique complaisante de la minorité des activistes musulmans et de leurs revendications accrédite et perpétue les préjugés islamophobes. La confusion entre la majorité silencieuse des musulmans français d'une part et ses minorités actives, porte-parole à la représentativité pour le moins discutable $^{(14)}$ est le point névralgique entretenant l'incompréhension mutuelle et suscitant l'hostilité. Les caricatures les plus dangereuses pour la société française ne sont pas toujours celles que l'on croit... 